\title{
Low sero-prevalence of hepatitis delta antibodies in HIV/ hepatitis B co-infected patients attending an urban HIV clinic in Uganda.
}

\author{
Elizabeth Katwesigye ${ }^{1}$, Emmanuel Seremba ${ }^{2}$, Fred Semitala ${ }^{1}$, Ponsiano Ocama ${ }^{1}$
}

\author{
1. Department of Medicine, Mulago Hospital and Makerere University College of \\ Health Sciences, Kampala, Uganda \\ 2. Department of Medicine, Mulago Hospital, Kampala, Uganda
}

\begin{abstract}
Background: Co-infection with hepatitis B (HBV) and hepatitis D (HDV) is common among human immunodeficiency virus (HIV) infected individuals in developing countries and it aggressively accelerates progression of liver disease to cirrhosis and other complications. There is scarcity of data on HDV in sub-Saharan Africa. We investigated the sero-prevalence and factors associated with HDV antibody among HIV/HBV co-infected patients attending a large urban HIV clinic in Uganda.

Methods: We screened $189 \mathrm{HIV} / \mathrm{HBV}$ co-infected individuals for anti-HDV immunoglobulin G (IgG) and performed logistic regression to determine the associated factors. Socio-demographic, clinical data, immunological status, and liver fibrosis (as determined by the Aspartate transaminase to platelet ratio index and transient elastography) were included.

Results: Participants were predominately young and of sound immunologic status (median age 40 years, median CD4 440 cells/ $\mu \mathrm{l}) .98 \%$ were on ART regimens containing anti-HBV active medications $(95.2 \%$ were on TDF/3TC while $4.8 \%$ on $3 \mathrm{TC}$ containing regimen). Median duration on ART was 36 months (IQR 22-72). Anti-HDV was detected in 6/198, 3.2\% (95\% CI 1.14-6.92\%), associated with male gender and a duration of more than 5 years since HIV diagnosis.

Conclusions: The sero-prevalence of HDV antibodies among the HIV/HBV co-infected patients is low in a Ugandan urban cohort.

Keywords: Co-infection in Uganda, hepatitis delta antibodies, hepatitis B virus, HIV.

DOI: https://dx.doi.org/10.4314/ahs.v17i4.4

Cite as: Katwesigye E, Seremba E, Semitala F, Ocama P. Low sero-prevalence of hepatitis delta antibodies in HIV/ hepatitis B co-infected patients attending an urban HIV clinic in Uganda. Afri Health Sci.2017;17(4): 974-978. bttps:/ / dx.doi.org/ 10.4314/abs.v17i4.4
\end{abstract}

\section{Introduction}

Hepatitis delta virus (HDV) is a defective virus and depends on the hepatitis B surface antigen ( $\mathrm{HBsAg}$ ) for its existence ${ }^{1-3}$. Worldwide approximately 15-20 million people have been exposed to HDV infection, which approximately represents $5 \%$ of the population of chronic hepatitis B.. Hepatitis D virus infection results in the most aggressive form of chronic viral hepatitis especially in HIV infected persons ${ }^{4,5}$. Triple infection with HDV/ $\mathrm{HBV}$ and human immune deficiency virus (HIV) is common due to shared modes of transmission; mainly through unprotected sexual intercourse and exposure to

\section{Corresponding author: \\ Elizabeth Katwesigye, Department of Medicine, Makerere University College of Health Sciences P.O. Box 7072, Kampala Uganda \\ Tele: +256782770647 \\ Email: katwesigyee@gmail.com}

contaminated blood products ${ }^{6,7}$. Current treatment options for HIV/HBV have a limited effect on HDV infection therefore not be adequate if there is HDV coinfection as response to HDV will not be achieved and liver disease progression continues to occur ${ }^{8-12}$. There is scarcity of data on HDV in Uganda. To the best of our knowledge there has only been one study which reported HDV antibody prevalence of $30.6 \%$ in the HBsAg positive population and $3.1 \%$ in the general population in the Northern part of Uganda ${ }^{13}$. The aim of this study was to measure the prevalence and to ascertain the factors associated with hepatitis D antibodies among HIV/ HBV co-infected adults individuals in Uganda.

\section{Methods}

This cross sectional study was performed at the Infectious Diseases Institute clinic (IDI), Makerere University, Uganda located within Mulago Hospital complex. Ever since it was opened in 2002, this clinic has registered over 10,000 individuals of whom 8,300 are active and are retion License (https://creativecommons.org/licenses/by/4.0), which permits unrestricted use, distribution, and reproduction in any medium, provided the original work is properly cited.
} 
ceiving ART. Over the last two years, routine screening of HIV-infected clients for hepatitis B virus has been ongoing and there $250 \mathrm{HIV} / \mathrm{HBV}$ co-infected patients attending the IDI clinic.

Between September 2015 and February 2016, we were able to recruit 189 participants. Those that provided written informed consent had their CD4 T-cell count, clinical history relevant to HIV and HBV diagnosis and medication use focusing on ART abstracted from the clinic records. This information was supplemented by a data collection tool that captured socio demographic characteristics and risk factors of HDV transmission. Participants had a physical examination focusing on liver disease and a complete blood count and liver enzyme assays done. In addition, all the participants underwent liver fibrosis assessment using non-invasive approaches; the Aspartate transaminase to platelet ratio index (APRI) and transient elastography with the aim of comparing liver disease severity between the HDV and non HDV infected. We defined liver fibrosis as an APRI score of $\geq 1.5$ and transient elastography score of $\geq 9 \mathrm{kpa}$. Serum was tested for HDV IgG antibodies using the HDV IgG ELISA assay kit (AccuDiag ${ }^{\text {TM }}$ HDV-IgG ELISA, Diagnostic automation, inc. Woodland Hills, CA 91367, USA). The manufacturer reports $100 \%$ sensitivity and specificity for detecting HDV IgG antibodies using this test.

The study obtained ethical approval from Department of Medicine, School of Medicine Research and Ethics Committee (SOMREC), Makerere University, College of Health Sciences and the IDI scientific review committee.

\section{Statistics}

Data collected was analyzed using STATA software package version 11. Logistic regression was performed to determine the factors associated with anti-HDV. A P-value of $<0.05$ was considered to be significant.

\section{Results}

A convenient sample of $189 \mathrm{HIV} / \mathrm{HBV}$ co-infected patients was recruited. The study population was composed mainly of young individuals; median age of 40 (IQR 3346) years and of sound immunological status (median CD4 440 (IQR 155-590 cells/yl). Ninety-eight percent were on ART regimens that contained anti-HBV active medication ( $95.2 \%$ were on TDF/3TC while $4.8 \%$ on 3TC containing regimen, $2 \%$ were not on ART). Median duration on ART was 36 months (IQR 22-72). The majority $(56 \%)$ had documented HIV infection for more than 5 years and over two-thirds had been diagnosed with HBV co-infection at least two years prior to this study (table 1).

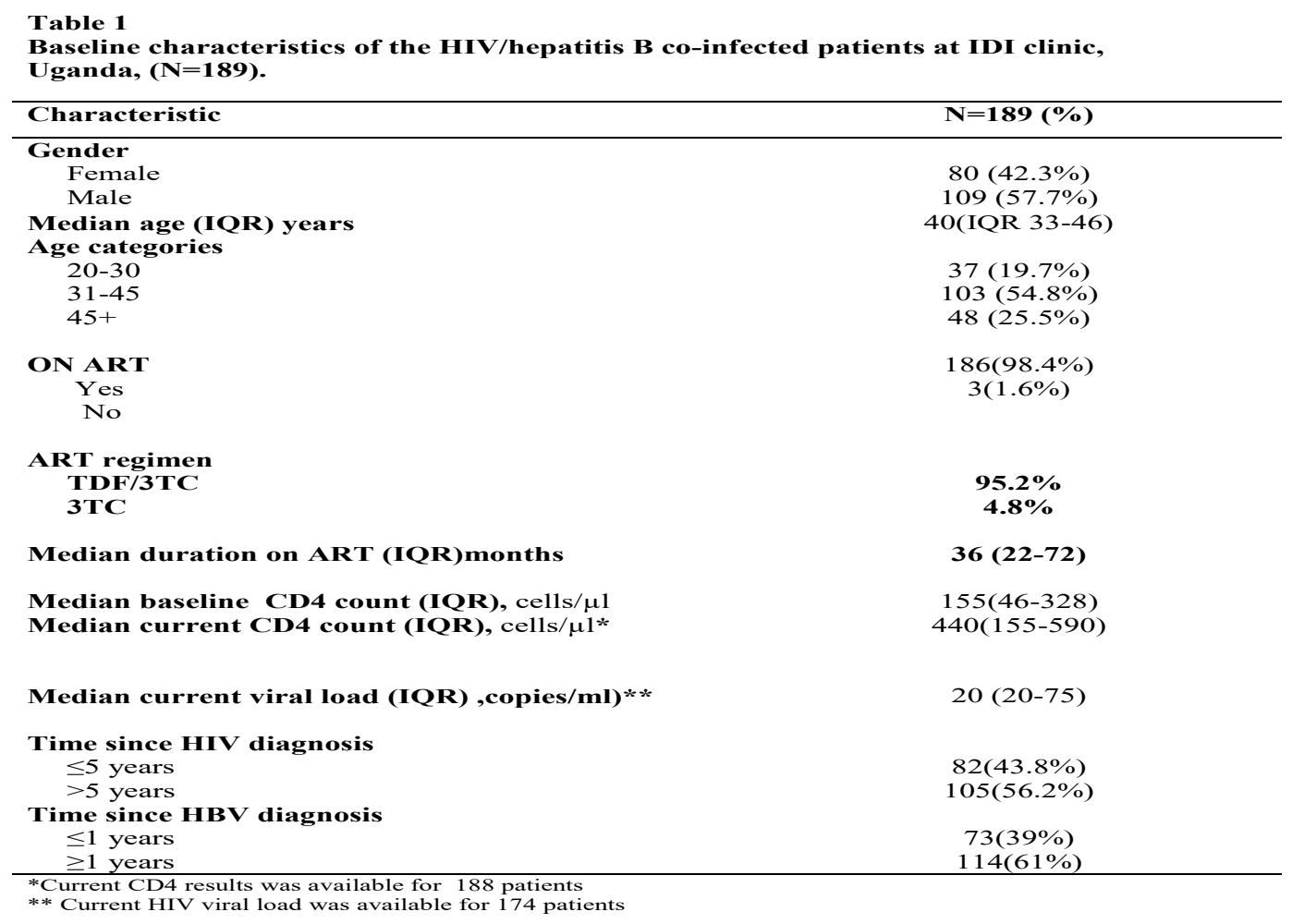


The prevalence of hepatitis delta antibody was 3.2\% (95\% confidence interval $1.42-6.92 \%$ ). All the participants that tested positive for hepatitis delta antibodies were male. They had been diagnosed with HIV and started on ART at least five years prior to this study (table 2).

The liver enzyme levels (ALT,AST), and the platelet count did not significantly differ among the individuals with positive or negative for anti-HDV, neither was there a difference in the HIV viral load and CD4 T-cell count among individuals in these categories.

Furthermore, there was no evidence of liver fibrosis among the individuals that tested positive for anti HDV antibody (normal APRI SCORE $(<1$ ), and 5/6 had a transient elastography score of $<7 \mathrm{kpa})$. One of the participants had invalid fibroscan test. Among the HDV negative participants, liver fibrosis was present in $5 \%$ by the APRI score and $20 \%$ by transient elastography score.

\section{Discussion}

Our study demonstrated a prevalence of hepatitis delta antibodies in HIV/HBV co infected patients in an urban HIV clinic in Uganda of 3.2\%. These results are comparable to some other studies in sub-Saharan Africa (SSA) done in similar settings ${ }^{14-16}$ but quite different in other selected sub-populations including those with chronic liver disease in West Africa where a high anti-HDV prevalence ranging from 12.2 to $81.7 \%$ was documented ${ }^{17}$. Also other studies in Africa have showed varying prevalence rates of HDV ranging from $0-50 \%$ in different countries ${ }^{18-20}$. Similarly in the developed countries, high anti-HDV prevalence's of $10-15 \%$ have been documented in recent studies ${ }^{1,21,22}$. Reasons for the variations in the prevalence of HDV exposure or infection are not clear. This could be a result of modes of transmission whereby in some of these countries there could be higher rates of IVD use $^{21,22}$.

In addition, we have confirmed earlier findings that male gender was significantly associated with anti HDV antibody positivity ${ }^{23,24}$.

Within the limits of small numbers in our study, we observed that living with HIV for five or more years was also significantly associated with HDV antibodies despite anti retro viral (ART) use. This data may suggest that ART may not affect HDV antibodies however a larger study is required to assess the impact of ART on HDV infection ${ }^{8}$. However, HIV may increase the risk of HDV acquisition since immunosuppressive states increase the likelihood of chronic hepatitis B which serves as a buffer to hepatitis D acquisition.

This study did not demonstrate significant differences in the liver fibrosis scores among the HDV negative and positive patients, which is in agreement with the clinical and laboratory data that did not suggest that individuals with detectable anti-HDV had a chronic liver disease. However, it may also be possible that the results reflect false positive test for HDV since no confirmation was made using HDV viral loads. On the other had it could also be a result of resolved infection. These have been demonstrated in others studies as well in African settings ${ }^{14}$.

\section{Limitations}

Our study has some limitations. We used a convenient sample of $\mathrm{HIV} / \mathrm{HBV}$ co-infected individuals that we could access. The few numbers of anti HDV antibody positive individuals identified in this study limited our ability to ascertain the factors associated with triple infection. However, given that similar findings had been recorded elsewhere in East Africa, our findings are likely to be valid. Furthermore, we were unable to perform HDV RNA to confirm current hepatitis delta infection and hence this study was based on HDV exposure and not necessarily current infection.

\section{Conclusion}

The sero prevalence of HDV antibodies among the HIV / HBV co-infected patients is very low in a Ugandan urban cohort as compared with developed countries where intravenous drug use is common. Routine screening for anti-HDV among individuals with $\mathrm{HIV} / \mathrm{HBV}$ co-infection in this setting may not be cost effective, however in absence of a known etiology, targeted testing can be done for patients with liver disease if not improving or developing liver disease while on a tenofovir based-ART regimen.

Following this pilot, a larger study in which HDV-RNA testing is performed is recommended to assess with better accuracy the magnitude of HDV infection in Uganda.

\section{Acknowledgements}

The authors would like to acknowledge the Infectious Diseases Institute clinic staff and patients for the participation in this study. Support for research was provided by Fogarty International Centre, National Institutes of 
Health (grant \# D43TW009771 "HIV co-infections in Uganda: TB, Cryptococcus and Viral Hepatitis.

\section{Conflict of interest}

The authors declare no conflict of interest

\section{Author's contributions}

EK, ES, FS and PO participated in the conception of the study, ES, FS and PO assisted in editing of the proposal. EK collected all the data and drafted the manuscript. ES, FS and PO helped with review of the manuscript. All authors approved the final manuscript

\section{References}

1. Hughes SA, Wedemeyer H, Harrison PM. Hepatitis delta virus. The Lancet. 2011;378(9785):73 -85.

2. Rizzetto M, Ciancio A, editors. Epidemiology of hepatitis D. Seminars in liver disease; 2012: Thieme Medical Publishers.

3. Tahaei SME, Mohebbi SR, Azimzadeh P, Behelgardi A, Sanati A, Mohammadi P, et al. Prevalence of hepatitis $\mathrm{D}$ virus in hepatitis $\mathrm{B}$ virus infected patients referred to Taleghani hospital, Tehran, Iran. Gastroenterology and hepatology from bed to bench. 2014;7(3):144.

4. Palella Jr FJ, Baker RK, Moorman AC, Chmiel JS, Wood KC, Brooks JT, et al. Mortality in the highly active antiretroviral therapy era: changing causes of death and disease in the HIV outpatient study. JAIDS Journal of Acquired Immune Deficiency Syndromes. 2006;43(1):27-34.

5. Calle SB, Manns MP, Wedemeyer H, editors. Hepatitis delta and HIV infection. Seminars in liver disease; 2012.

6. Wedemeyer H, Manns MP. Epidemiology, pathogenesis and management of hepatitis D: update and challenges ahead. Nat Rev Gastroenterol Hepatol. 2010;7(1):31-40.

7. Liaw Y-F, Chiu K-W, Chu C-M, Sheen I-S, Huang M-J. Heterosexual transmission of hepatitis delta virus in the general population of an area endemic for hepatitis B virus infection: a prospective study. Journal of Infectious Diseases. 1990;162(5):1170-2.

8. Boyd A, Lacombe K, Miailhes P, Gozlan J, Bonnard P, Molina JM, et al. Longitudinal evaluation of viral interactions in treated HIV-hepatitis B co-infected patients with additional hepatitis $\mathrm{C}$ and $\mathrm{D}$ virus. Journal of viral hepatitis. 2010;17(1):65-76.

9. Heidrich B, Manns M, Wedemeyer H. Treatment Options for Hepatitis Delta Virus Infection. Current Infectious Disease Reports. 2013;15(1):31-8.

10. Niro G, Ciancio A, Tillman H, Lagget M, Olivero A,
Perri F, et al. Lamivudine therapy in chronic delta hepatitis: a multicentre randomized-controlled pilot study. Alimentary pharmacology \& therapeutics. 2005;22(3):227 -32. 11. Farci P, Mandas A, Coiana A, Lai ME, Desmet V, Van Eyken $\mathrm{P}$, et al. Treatment of chronic hepatitis D with interferon alfa-2a. New England Journal of Medicine. 1994;330(2):88-94.

12. Soriano V, Vispo E, Sierra-Enguita R, de Mendoza C, Fernández-Montero JV, Labarga P, et al. Efficacy of prolonged tenofovir therapy on hepatitis delta in HIV-infected patients. Aids. 2014;28(16):2389 -94.

13. de Lalla F, Rizzardini G, Rinaldi E, Santoro D, Zeli PL, Verga G. HIV, HBV, delta-agent and Treponema pallidum infections in two rural African areas. Transactions of the Royal Society of Tropical Medicine and Hygiene. 1990;84(1):144-7.

14. Winter A, Letang E, Kalinjuma AV, Kimera N, Ntamatungiro A, Glass T, et al. Absence of hepatitis delta infection in a large rural HIV cohort in Tanzania. International Journal of Infectious Diseases. 2016;46:8-10.

15. Diop-Ndiaye H, Touré-Kane C, Etard J-F, Lo G, Diaw P, Ngom-Gueye N, et al. Hepatitis B, C seroprevalence and delta viruses in HIV-1 Senegalese patients at HAART initiation (retrospective study). Journal of medical virology. 2008;80(8):1332-6.

16. Onyekwere C, Audu R, Duro-Emmanuel F, Ige F. Hepatitis D infection in Nigeria. Indian Journal of Gastroenterology. 2012;31(1):34-5.

17. Andernach IE, Leiss LV, Tarnagda ZS, Tahita MC, Otegbayo JA, Forbi JC, et al. Characterization of Hepatitis Delta Virus in Sub-Saharan Africa. Journal of clinical microbiology. 2014;52(5):1629-36.

18. Bekondi C, Mobima T, Ouavene J, Koffi B, Konamna $\mathrm{X}$, Béré A, et al. [Etiopathological factors of hepatocellular carcinoma in Bangui, Central African Republic: clinical, biological characteristics and virological aspects of patients]. Pathologie-biologie. 2010;58(2):152 -5.

19. Lunel-Fabiani F, Mansour W, Amar AO, Aye M, Le Gal F, Malick FZF, et al. Impact of hepatitis B and delta virus co-infection on liver disease in Mauritania: A cross sectional study. Journal of Infection. 2013;67(5):448-57.

20. Cunha L, Plouzeau C, Ingrand P, Gudo JPS, Ingrand I, Mondlane J, et al. Use of replacement blood donors to study the epidemiology of major blood-borne viruses in the general population of Maputo, Mozambique. Journal of medical virology. 2007;79(12):1832-40.

21. Soriano V, Grint D, Monforte AdA, Horban A, Leen C, Poveda E, et al. Hepatitis delta in HIV-infected indi- 
viduals in Europe. Aids. 2011;25(16):1987 -92.

22. Hung C-C, Wu S-M, Lin P-H, Sheng W-H, Yang Z-Y, Sun H-Y, et al. Increasing Incidence of Recent Hepatitis $\mathrm{D}$ Virus Infection in HIV-Infected Patients in an Area Hyperendemic for Hepatitis B Virus Infection. Clinical Infectious Diseases. 2014;58(11):1625-33.

23. Fernández-Montero JV, Vispo E, Barreiro P, Sier-
ra-Enguita R, de Mendoza C, Labarga $P$, et al. Hepatitis Delta Is a Major Determinant of Liver Decompensation Events and Death in HIV-Infected Patients. Clinical Infectious Diseases. 2014;58(11):1549-53.

24. Shaikh MA, Shaikh WM, Solangi GA, Shaikh BA, Soomro MA. Frequency of hepatitis D virus infection in hepatitis B surface antigen-positive liver diseases. J Coll Physicians Surg Pak. 2011;21(1):23-5. 